\title{
Perivascular Lymphocyte Clusters Induced by Gastric Subserous Layer Vaccination Mediate Optimal Immunity against Helicobacter through Facilitating Immune Cell Infiltration and Local Antibody Response
}

\author{
Chupeng Hu, ${ }^{1,2}$ Wei Liu, ${ }^{1,2}$ Ningyin Xu, ${ }^{1,2}$ An Huang, ${ }^{1,2}$ Zhiqin Zeng, ${ }^{1,2}$ Shuanghui Luo, ${ }^{1,2}$ \\ Zhenxing Zhang, ${ }^{1,2}$ Menghui Fan, ${ }^{1,2}$ Feng Ye, ${ }^{3}$ Tao Xi $\mathbb{D}^{1,2}$ and Yingying Xing $\mathbb{D}^{1,2}$ \\ ${ }^{1}$ School of Life Science and Technology, China Pharmaceutical University, Nanjing 210009, China \\ ${ }^{2}$ Jiangsu Key Laboratory of Carcinogenesis and Intervention, China Pharmaceutical University, Nanjing 210009, China \\ ${ }^{3}$ Department of Gastroenterology, The First Affiliated Hospital of Nanjing Medical University, Nanjing, China
}

Correspondence should be addressed to Tao Xi; xitao18@hotmail.com and Yingying Xing; cpuskyxyy@126.com

Received 15 July 2019; Accepted 19 December 2019; Published 11 January 2020

Academic Editor: Benoit Stijlemans

Copyright (c) 2020 Chupeng Hu et al. This is an open access article distributed under the Creative Commons Attribution License, which permits unrestricted use, distribution, and reproduction in any medium, provided the original work is properly cited.

\begin{abstract}
Background. In situ vaccination-induced local inflammatory response resulted in the establishment of a pool of tissue-resident memory $\mathrm{T}\left(\mathrm{T}_{\mathrm{RM}}\right)$ cells and new vessels after the resolution of inflammation. $\mathrm{T}_{\mathrm{RM}}$ cells have received increasing attention; however, the role of new vessels in protective response is still unknown. Materials and Methods. We performed the laparotomy to access the stomach and injected alum-based vaccine into the gastric subserous layer (GSL). At 28 days post vaccination, a parabiosis mouse model along with depletion of anti-CD90.2 antibody was employed to explore the function of perivascular lymphocyte clusters in recall responses. The composition of the gastric lymphocyte clusters was analyzed by immunofluorescence staining. Antibody responses were detected using ELISA. Gastric lymphocytes were analyzed using flow cytometry. Results. GSL vaccination induced the formation of new vessels in the inflamed region. These new vessels were different from native vessels in that they were generally accompanied by perivascular lymphocyte clusters that mainly consisted of CD90-expressing cells. Additionally, histological analysis revealed the presence of $\mathrm{CD} 4^{+}$and $\mathrm{CD} 8^{+} \mathrm{T}$ cells in the perivascular lymphocyte clusters. Administration of a dose of an anti-CD90.2 antibody to GSL-vaccinated mice resolved these clusters. The efficacy of protection was compared in the parabiosis mice. Upon challenge, the presence of perivascular lymphocyte clusters was responsible for the fast recall response, as depletion of these clusters by CD90.2 antibody administration resulted in decreased expressions of VCAM-1, Madcam-1, and TNF- $\alpha$, as well as lower recruitment of proinflammatory immune cells, decreased antibody levels, and poor protection. Conclusions. Our research demonstrates that in situ vaccination-induced regional inflammatory response contributes to optimal recall response not only by establishing a $\mathrm{CD} 4^{+} \mathrm{T}_{\mathrm{RM}}$ pool but also by creating an "expressway," i.e., perivascular lymphocyte cluster.
\end{abstract}

\section{Introduction}

In situ vaccination-induced local inflammatory response induced not only the establishment of a tissue-resident memory $\mathrm{T}\left(\mathrm{T}_{\mathrm{RM}}\right)$ cell pool $[1,2]$ but also the formation of new vessels [3]. Circulating immune cells generally permeate across postcapillary venule and infiltrate into the mucosa to induce protection against mucosal pathogens [4]. It remained unclear whether these vessels played a role in protective response.

In a previous study, we used vaccine composed of aluminum adjuvant and CCF (CCF, a recombinant Helicobacter pylori subunit vaccine, a dual-antigen epitope, and a dualadjuvant vaccine constructed as previously described [5]), to vaccinate the gastric subserous layer (GSL) of mice [6]. GSL vaccination-induced mucosal inflammation in the 
stomach and subsequently a pool of $\mathrm{CD} 4^{+}$tissue-resident $\mathrm{T}$ cells $\left(C D 4^{+} \mathrm{T}_{\mathrm{RM}}\right)$ were established in the epithelium. After in situ injection with $5 \mu \mathrm{L}$ vaccine in the GSL, acute inflammation was observed at the mucosa around the vaccination site. Interestingly, upon challenge, circulating immune cells preferentially migrated towards the infected epithelium through vessels, which were located in the vaccination region (Supplementary Figure 1). It is noteworthy that new blood vessels are prevalent in inflammationexperienced tissue $[1,7,8]$. We speculated that they may play a role that distinguished them from the native vessels. Perivascular infiltration of DC-T cell clusters was formed at antigen-challenged sites, which enhanced proliferation and reactivation of effector $\mathrm{T}$ cells through integrin LFA-1 and local antigen exposure [9]. Additionally, perivascular infiltration of lymphocytes was associated with recruitment of myeloid immune cells and lymphocytes to peripheral tissue in a manner dependent on adhesion molecules or cytokines [9-11]. One of the patterns was classical inflammation-induced activation of endothelial cells through secreting VCAM-1 or Madcam-1 and various cytokines [12-14]. As described above, perivascular leukocyte clusters play an important role in the progression of inflammatory response. In this study, to highlight the need for in situ vaccination, we investigated the role of new vessels with perivascular lymphocyte clusters that were generated in in situ vaccination-induced inflammation in recall response.

\section{Material and Method}

2.1. Reagents. Alum adjuvant was purchased from Thermo Company. Fetal bovine serum (FBS) was purchased from Gibco Laboratories (Grand Island, NY, USA). ChamQ ${ }^{\mathrm{TM}}$ SYBR qPCR master mix (high ROX premixed) was obtained from Vazyme Biotech Co., Ltd. (Nanjing, China). 3,3,5,5Tetramethylbenzidine (TMB) solution was purchased from Solarbio. TRIzol reagent was procured from Invitrogen. In vivo Mab anti-mouse $\mathrm{CD} 90.2$ and $\mathrm{IgG}_{2 \mathrm{~b}}$ isotype were obtained from Bio X cell. All other solvents and chemicals were of analytical grade and used without further purification.

2.2. Vaccine Preparation. The preparation and storage of purified CCF protein, a dual-antigen epitope and dualadjuvant vaccine constructed by cholera toxin $\mathrm{B}$, multiepitopes from $\mathrm{H}$. pylori urease, and self-adjuvant regions from S. typhimurium phase I flagellin FliC, called CTBUE-CF (CCF), were performed in previous protocols [15]. Briefly, the CCF protein was expressed by Escherichia coli Rosetta (DE3) cells with pET-28a-CCF. The protein was first purified using nickel affinity chromatography (GE Healthcare), followed by anion-exchange chromatography with DEAE Sepharose FF [16] (Amersham Pharmacia Biotech AB, Sweden). The purity of CCF was confirmed using the Coomassie blue staining. Vaccine with alum was prepared with equal volumes of CCF solution and alum adjuvant.
2.3. Animals. Female 6 to 8 -week-old C57BL/6 mice were purchased from the Comparative Medicine Center of Yangzhou University and housed under specific pathogen-free conditions in the Animal Experimental Center of China Pharmaceutical University. All animal experiments were approved by the Animal Ethical and Experimental Committee of the China Pharmaceutical University.

2.4. Gastric Subserous Layer Vaccination. The 6 to 8-weekold female C57BL/6 mice were anesthetized using intraperitoneal injection with a mixture of $100 \mathrm{mg} / \mathrm{kg}$ ketamine and $15 \mathrm{mg} / \mathrm{kg}$ xylazine; the experiment was conducted under aseptic conditions, and mice were placed on the thermostatic hot plate. After shaving off around the right abdomen, a $1 \mathrm{~cm}$ wide skin incision was made above the location of the stomach. After simple celiotomy laparotomy, the stomach was exposed using Forceps, and the gastric subserous layer of the greater curvature was injected with $5 \mu \mathrm{L}$ vaccine preparation using a microsyringe with a $33 \mathrm{G}$ needle. Next, the peritoneal incision was uninterruptedly sutured with 7-0 PGA absorbable suture, and the skin incision was performed using interrupted sutures (Shanghai Pudong Jinhuan Medical Products Co., Ltd.).

2.5. Parabiosis Mouse Pair Surgery. Mice were cohoused for 2 weeks prior to jointed surgery [17]. The experiment was performed under aseptic conditions. Naïve and immunized mice were anesthetized using intraperitoneal injection with a mixture of $100 \mathrm{mg} / \mathrm{kg}$ ketamine and $15 \mathrm{mg} / \mathrm{kg}$ xylazine. After shaving the corresponding lateral aspects of each mouse, a matching incision was made on the lateral skin from the elbow to the hip. Then, the tibia and ulna of each mouse were bound together with 3-0 PGA nonabsorbable suture (Shanghai Pudong Jinhuan Medical Products Co., Ltd.) to join the forelimbs and hind limbs together; the matching skin incision of each mouse was sutured together using 5-0 PGA absorbable suture (Shanghai Pudong Jinhuan Medical Products Co., Ltd.) to induce angiogenesis for a shared circulation between two mice.

2.6. Preparation of Single Cell Suspensions from Gastric Mucosa. The single cell suspensions were prepared similarly as previously described $[15,18]$. In brief, the whole stomach of mice was cut and isolated using lesser curvature, prior to being removed; the content was placed into $15 \mathrm{~mL}$ RPMI1640 containing $10 \mathrm{mM}$ HEPES, 10\% PBS, $4 \mathrm{mM}$ EDTA, and $0.5 \mathrm{mM}$ DTT. Gastric epithelial lymphocytes were isolated by shaking at $250 \mathrm{rpm}$ and $37^{\circ} \mathrm{C}$ for $30 \mathrm{~min}$. The single cell suspension was obtained by passing it through a $70 \mu \mathrm{m}$ cell strainer. After washing and centrifugation, cell pellets were resuspended in an appropriate medium for further analysis or culture.

2.7. Preparation of Single Cell Suspensions from the Blood. The blood was isolated from mice and resuspended with $5 \mathrm{~mL}$ erythrocyte lysis buffer (BioLegend) and washed twice with $10 \mathrm{~mL}$ PBS containing 5\% FBS. The cells were then collected for FACS analysis. 
2.8. Antigen-Specific $\mathrm{CD}^{+}{ }^{+} \mathrm{T}$ Cell Analysis. Antigen-specific $\mathrm{CD} 4^{+} \mathrm{T}$ cells were prepared as previously described [6]. Single cell suspensions from the stomach were purified with $67 \% / 44 \%$ Percoll gradients. The cells at the interface were collected and washed with $7 \mathrm{~mL}$ RPMI 1640 containing $10 \%$ FBS. To detect Ag-specific $\mathrm{CD}^{+}{ }^{+} \mathrm{T}$ cells, purified single cell suspensions from the stomach were stimulated for $12 \mathrm{~h}$ with $1 * 10^{6}$ naïve CFSE-labeled splenocytes that were preloaded with CCF in RPMI 1640 containing 10\% FBS and $5 \mu \mathrm{g} / \mathrm{mL}$ Brefeldin A. After collection, the cells were stained for intracellular cytokines.

2.9. FACS Analysis. Single cell suspensions from the blood and gastric tissue were stained with the following antibodies: anti-CD45 (30-F11), anti-CD3e (145-2C11), anti-CD90.2 (30-H12), anti-CD4 (GK1.5 or RM4-4), anti-CD11b (M1/70), anti-CD8 $\alpha$ (53-6.7), anti-CD19 (6D5), anti-MHC class II (M5/114.15.2), anti-Ly6C (HK1.4), anti-Gr-1 (RB6$8 \mathrm{C} 5$ ), and anti-CD11c (N418) purchased from BioLegend or BD Pharmingen. All the samples were incubated for 20 min on ice in the dark. Multiparameter analyses were performed on a BD FACS Aria II or a BD FACSCalibur flow cytometer.

2.10. Immunofluorescent Staining. Frozen sections of gastric tissue $(20$ or $10 \mu \mathrm{m})$ were cut and dried at room temperature. After blocking in a 5\% bovine serum albumin PBS solution for $1 \mathrm{~h}$, these sections were stained with the Abs an Alexa Fluor $^{\circledR}$ 488-anti-CD4 (GK1.5, BioLegend) antibody and/or purified anti-CD11b (M1/70, BioLegend) or anti-CD8 $\alpha$ (53-6.7, BioLegend), purified anti-mouse I-A/I-E (BioLegend), Alexa Fluor ${ }^{\circledR} 488$-anti-CD90.2 (BioLegend), antiCD31 (BD Biosciences), and anti-VCAM-1 (P3C4, BioLegend) antibody followed by goat anti-rat IgG2a/IgG2b Alexa Fluor $^{\circledR}$ 488/594 antibody (BioLegend). The slides were washed, counterstained with DAPI to visualize cell nuclei, and analyzed with fluorescence microscopy. All images were acquired with a Panoramic 250 Flash III Scanner (3DHistech).

2.11. Quantification of Fluorescence Intensity. Fluorescence images were acquired with a Panoramic 250 Flash III Scanner (3DHistech); individual CD31 or VCAM-1 images were loaded into the ImageJ64 software as JPEG files. Color images were then converted into a binary scale, which transforms images into black and white pixels. Quantification was then performed on the black pixels using the "analyze particle" function. The mean fluorescence intensity in a random region was calculated.

2.12. Quantitative RT-PCR. Total RNA was extracted from gastric tissue using TRIzol reagent (Invitrogen), and each RNA sample was reverse-transcribed into cDNA using MMLV (Promega, USA) and following standard protocols. Quantitative real-time PCR was performed with a conventional TaqMan SYBR green-based quantification method. The expressed genes in gastric tissues were quantitated using RT-qPCR and the set of primers that were designed by Sangon Biotech Co., Ltd. (Shanghai, China) based on the sequence numbers found in Supplementary Table 1.
2.13. Measurement of Antigen-Specific Antibody in the Serum. The blood was obtained from the mouse angular vein for the detection of the levels of antigen-specific antibody using ELISA as follows: the 96-well ELISA plates (Corning Laboratories, Corning, NY) were coated overnight at $4^{\circ} \mathrm{C}$ with $100 \mu \mathrm{L}$ of $10 \mu \mathrm{g} / \mathrm{mL}$ CCF. $100 \mu \mathrm{L}$ of $1: 100$ diluted serum was added into the plates and then incubated at $37^{\circ} \mathrm{C}$ for $1 \mathrm{~h}$. Then $100 \mu \mathrm{L}$ of diluted HRP-conjugated goat antimouse IgG1 and IgM (Santa Cruz, diluted 1:2000) was added as the second antibody and then incubated at $37^{\circ} \mathrm{C}$ for $1 \mathrm{~h}$. The color reaction was performed through adding the substrate 3,3,5,5-tetramethylbenzidine (TMB) solution (Solarbio). The absorbance value was detected at $450 \mathrm{~nm}$ using a plate reader. Each sample was measured in triplicate.

2.14. Measurement of Antigen-Specific Secretory IgA Antibody in Gastric Tissue. The supernatant from the gastric tissue was obtained by homogenizing with a blade-blender homogenizer, and $100 \mu \mathrm{L}$ diluted supernatant in the proportion of $1: 5$ was added into the 96 -well plates. HRP-conjugated goat anti-mouse IgA was performed to detect the sIgA. Other steps were carried out following the measurement of the antigen-specific antibody in the serum.

2.15. CD $90.2^{+} \mathrm{T}$ Cell Depletion Experiments. Six to eightweek-old female C57BL/6 mice were immunized through gastric subserous layer vaccination. After 4 weeks, each mouse was administered with a dose of $500 \mu \mathrm{g}$ anti-CD90.2 $(30 \mathrm{H} 12)$ or anti-RatIgG2b (LTF-2) antibody each mice i.p. 2 times at 3-day intervals [19]. In vivo depletion was confirmed through FACS analysis of the cell suspension from the blood.

2.16. H. felis Challenge. H. felis strain (ATCC 49179) was cultured, as previously described [20], at $37^{\circ}$ Con interlayer of solid and liquid media both added with $7 \%$ heatinactivated fetal bovine serum and $10 \mu \mathrm{g} / \mathrm{mL}$ vancomycin under microaerophilic conditions. The bacteria were harvested through centrifugation and resuspended in fresh medium after 3-4 days. Prior to being inoculated with $0.5 \times 10^{9} \mathrm{H}$. felis in $200 \mu \mathrm{L}$ medium, the mice were fasted 8 hours without solid food and 4 hours without water. All the groups were challenged 2 times at 3-day intervals via p.o. After 1 week, tissues from these mice were harvested and analyzed.

2.17. Measurement of H. felis in the Gastric Mucosa. To detect the colonization of $\mathrm{H}$. felis in the gastric mucosa, qRT-PCR was performed to detect $H$. felis $16 \mathrm{~S}$ expression in the gastric tissue. The total DNA of gastric tissue was extracted with a Qiagen DNA Tissue Mini Kit according to the manufacturer's instructions. Quantitation was performed with the ChamQ Universal SYBR qPCR master mix. RT-qPCR was performed using a set of primers and was designed by Sangon Biotech Co., Ltd. (Shanghai, China) based on the sequence numbers found in Supplementary Table 1. Analysis and fold change were determined using the CT method.

2.18. Statistics Analysis. The results are presented as the mean \pm SD. Statistical analysis was performed using ANOVA 
or the $t$-test with the GraphPad Prism 6.0 software; values of $P<0.05$ were considered a significant statistical difference for all experiments.

\section{Result}

3.1. Formation of New Vessels with Perivascular Clusters at Inflammation-Experienced Gastric Tissue. Gastric subserous layer (GSL) vaccination with alum-based vaccine induced mucosa inflammation in the stomach, and subsequently, a pool of $\mathrm{CD}^{+}$tissue-resident $\mathrm{T}$ cells $\left(\mathrm{CD} 4^{+} \mathrm{T}_{\mathrm{RM}}\right)$ was established in the epithelium. Upon challenge, we found that a large number of circulating immune cells preferentially migrated to the infected epithelium through vessels (Supplementary Figure 1), which were located at the vaccination region. To estimate the perivascular lymphocyte clusters formed in the vaccination region, we carried out an experiment: We injected alum-based vaccine into the GSL of mice. Then 28 days post immunization, the mice were sacrificed, and the gastric tissue was collected for further test. First, when the whole stomach was dissected, we found a white lump at the site of vaccination (Figure 1(a)). Next, we sniped longitudinally the vaccination region and performed immunofluorescence staining of $\mathrm{CD}^{+} 1^{+}$vessels. Substantially increased numbers of vessels were observed in the vaccination site of mice compared with those in the naïve mice (Figures 1(b) and 1(c)). Immunofluorescence staining indicated that abundant CD90.2 cells infiltrated around vessels in the vaccination region. Noteworthy histological assay showed perivascular infiltration of $\mathrm{CD} 4^{+}$ $\mathrm{T}$ and $\mathrm{CD}^{+} \mathrm{T}$ cells, but not CD11b or MHCII-expressing cells emerged in the vessels of the vaccination region (Figure 1(d)). These findings led us to hypothesize that intensive accumulation of $\mathrm{CD}^{+} / \mathrm{CD}^{+} \mathrm{T}$ cells around vessels of the vaccination region contributes to the formation of perivascular lymphocyte clusters.

3.2. Perivascular Lymphocyte Clusters Contributed to Protection against $H$. felis Infection. Next, we set out to characterize the role of the perivascular lymphocyte clusters in protective response. A parabiosis mouse model was established. All mice underwent laparotomy to access the stomach, and an alum-based vaccine was into GSL. At day 28, these immunized mice were divided into two groups. For one group, half of the mice were administered with anti-CD90.2 antibody and cohoused with the other half. At day 42, the anti-CD90.2 antibody-treated mice and the other mice not injected with an antibody were surgically jointed. At day 56, the indicated partner was infected with $H$. felis (Figure 2(a)). Another group was treated the same way, but the IgG2b isotype was used. The establishment of a parabiosis model is aimed at sharing circulating CD90.2 cells between the antibody-treated mice and immunized mice. As expected, compared to that in the isotype-treated mice, the CD90.2 cells were depleted in the blood of the antiCD90.2 antibody-treated mice (Figure 2(b)). At 2 weeks post parabiosis, the number of circulating CD90.2 cells in the two groups of mice was similar (Figures 2(b) and 2(c)). Meanwhile, an immunolocalization assay was performed and the images indicated that the perivascular lymphocyte clusters were depleted (Figure 2(d)).

Next, the indicated mice were infected with $H$. felis (Figure 2(e)). Compared with the naïve mice, a significant decrease of $\mathrm{H}$. felis in anti-CD90.2 or isotype control mAbtreated mice was detected by QRT-PCR. However, compared with isotype-treated mice, the colonization of $\mathrm{H}$. felis in antiCD90.2 antibody-treated mice was significantly higher (Figure 2(f)). These data suggested that perivascular lymphocyte clusters participated in protection against $H$. felis.

\subsection{Perivascular Lymphocyte Clusters Accelerated Infiltration} of Immune Cell in Gastric Mucosa. To explore how the perivascular lymphocyte clusters participated in protection against $H$. felis, we investigated the gastric mucosal infiltration of immune cells. We employed a parabiosis mouse model and anti-CD90.2 antibody depletion experiment as described above (Figure 2(a)). At day 3 post last infection, immune cell subsets of gastric mucosa were analyzed by flow cytometry. In comparison to those in the isotype-treated mice, most immune cell types except monocytes and neutrophils (i.e., total $\mathrm{T}$ cells, $\mathrm{CD}^{+} \mathrm{T}$ cells, DCs and macrophages) were prominently reduced in the anti-CD90.2 antibody-treated mice (Figures 3(a)-3(c)). The population of antigen-specific $\mathrm{CD} 4^{+} \mathrm{T}$ cells in gastric mucosa was analyzed using the flow cytometry. The results showed a significant decrease in IFN- $\gamma$ and IL-17-producing $\mathrm{CD} 4^{+}$ $\mathrm{T}$ cells in the anti-CD90.2 antibody-treated mice $(3.2 \%$ and $3.6 \%$, respectively) compared with those in the control mice $(4.3 \%$ and $6.5 \%$, respectively) (Figures $3(\mathrm{~d})-3(\mathrm{~g})$ ). Similar results were observed in the immunofluorescence staining (Figures 3(h) and 3(i)). These data indicated that perivascular lymphocyte clusters contributed to mucosal infiltration of immune cells.

In addition, at day 3 after the last infection, immunolocalization assay showed that anti-CD90.2 antibody-treated mice displayed a reduced expression of VCAM-1 in vessels of the vaccination region compared to that of the isotypetreated mice (Figures 4(b) and 4(c)). Similarly, the mRNA levels of Madcam-1 and TNF- $\alpha$ in the whole stomach of the anti-CD90.2 antibody-treated mice were reduced (Figure 4(a)). These data showed that perivascular lymphocyte clusters were associated with increased expression of VCAM-1 and Madcam-1 in the whole stomach, which might accelerate the recruitment of immune cells into the gastric mucosa.

3.4. Perivascular Lymphocyte Clusters Facilitated AntigenSpecific IgA Antibody Production. To assess whether perivascular lymphocyte clusters around vessels played a role in access of antigen-specific antibody to gastric tissue, we employed a parabiosis mouse model and anti-CD90.2 antibody depletion experiment as described above. The measurements of antibodies were performed on the whole stomach and blood. At 3 days after the last infection, ELISA revealed that levels of the IgM and IgG1 in the blood did not differ (Figure 5(a)), while antigen-specific IgA in the gastric tissue were reduced in the anti-CD90.2 antibody-treated mice compared with those in the isotype-treated mice (Figure 5(b)). 


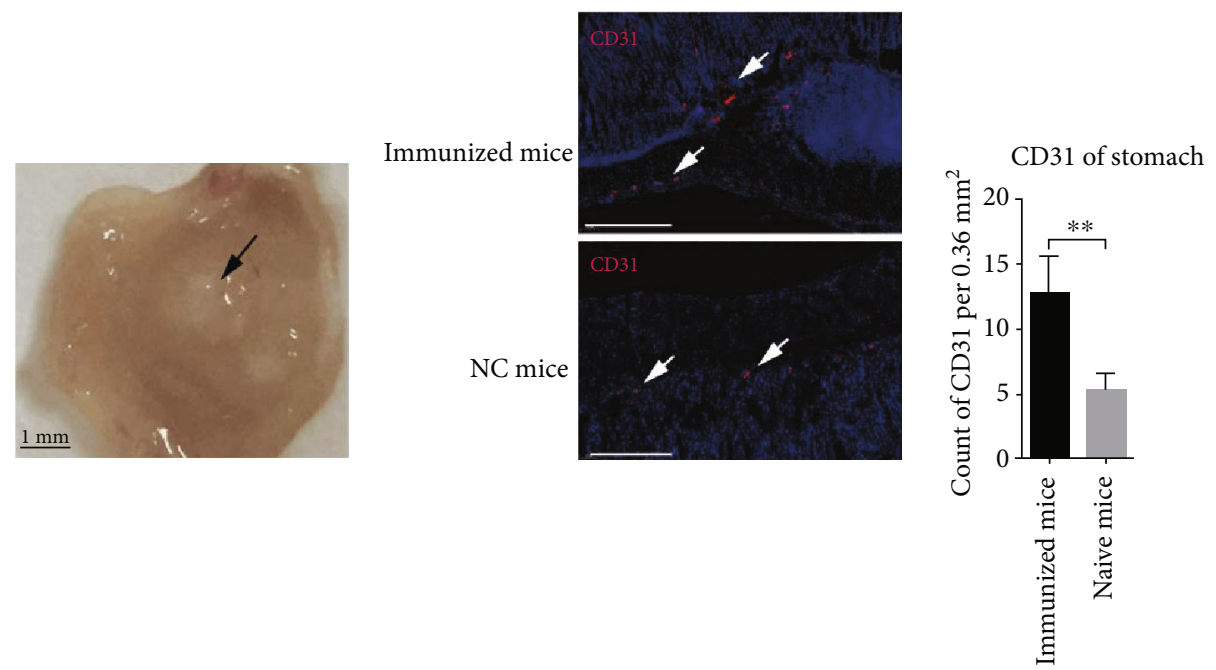

(a)

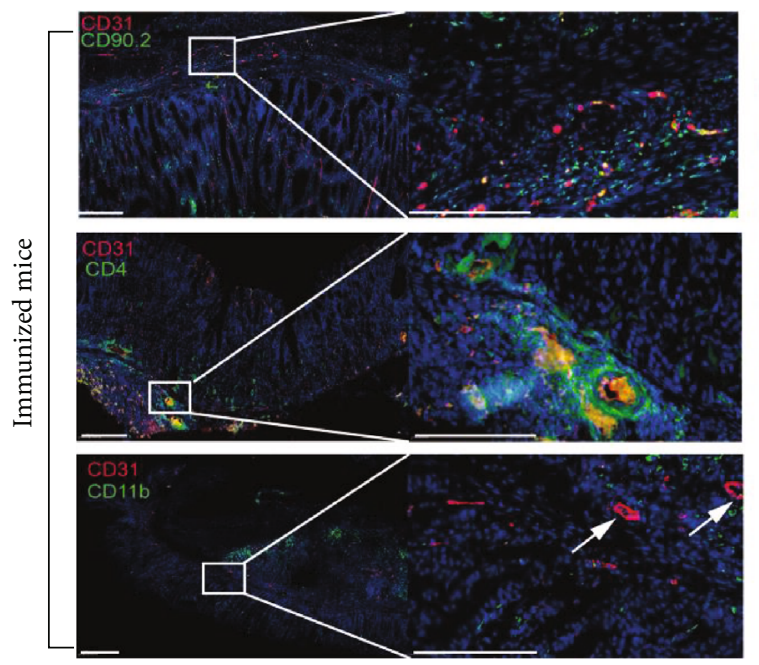

(b)

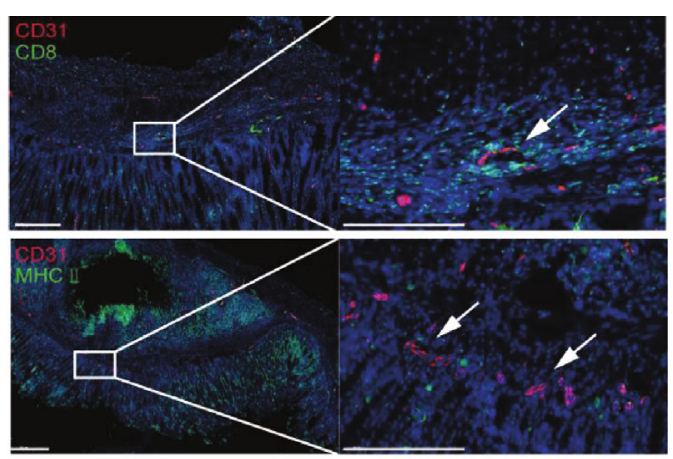

(d)

FIgURE 1: Formation of perivascular infiltrates of CD4/CD8 ${ }^{+} \mathrm{T}$ clusters in inflammation-experienced gastric subserous tissue. Six to eightweek-old female C57BL/6 mice were vaccinated in the gastric subserous layer. (a) 28 days later, the gastric tissue was exposed and a white lump was observed. (b, c) Frozen sections of gastric tissue from immunized or naïve mice were stained with antibodies against CD31 (red); nuclei are depicted by DAPI stained blue. Scale bars: $100 \mu \mathrm{m}$. The fluorescence images were acquired with a Panoramic 250 Flash III Scanner (3DHistech); counts of CD31 per $0.36 \mathrm{~mm}^{2}$ were randomly chosen and calculated. Values are the mean \pm SD $(n=3)$. ${ }^{* *} P<0.01$. (d) Frozen sections of gastric tissue from immunized mice were stained with antibodies against CD31 (red), CD90.2, CD4, CD8, CD11b, or MHCII (green); nuclei were depicted by DAPI stained blue. Scale bars: $200 \mu \mathrm{m}$.

After challenge at day 7, the antigen-specific IgM, IgG1, and IgA from gastric tissue were measured using ELISA. The result showed a higher expression of antigen-specific IgM, IgG1, and IgA in both anti-CD90.2 antibodytreated mice and isotype-treated mice compared with those in naive mice, while antigen-specific IgA in the gastric tissue were reduced in the anti-CD90.2 antibodytreated mice compared with those in the isotype-treated mice (Figures 5(c)-5(e)). Those results showed that antigen-specific IgA contributed to the early and later inflammation, but IgM and IgG1 participated in the later phase of gastric inflammation. Together, perivascular lymphocyte clusters were associated to infiltration of antigenspecific IgA antibody production in gastric tissue.

\section{Discussion}

Circulating immune cells (such as Th1/Th17 cells) permeate across postcapillary venules and infiltrate into the mucosa to induce protection against mucosal pathogens [4]. The role of Th1/Th17 cell-mediated post immunization gastritis is highlighted in the anti-Helicobacter pylori response [21,22]. Our primary experiment found that new vessels with perivascular lymphocyte clusters emerged in the vaccination region after resolution of GSL vaccination-induced inflammation. Circulating immune cells preferentially migrated to the infected epithelium through vessels. Here, we employed a parabiosis mouse model and anti-CD90 antibody depletion experiment to explore the role of perivascular lymphocyte 


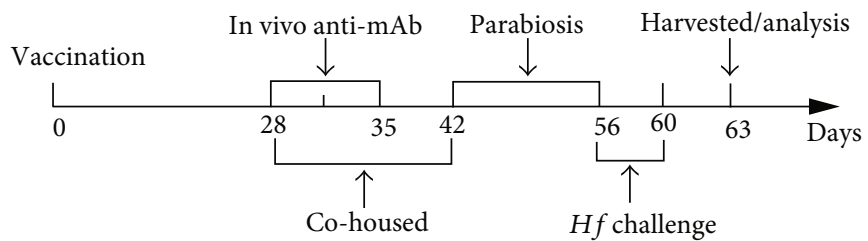

\begin{tabular}{lccc}
\multicolumn{4}{c}{ Parabiosis model } \\
\hline Group & Left partner & Right partner & H. felis challenge \\
\hline 1 & Immunized WT & Isotype immunized WT & Right \\
2 & Immunized WT & Anti-CD90.2 immunized WT & Right
\end{tabular}

(a)
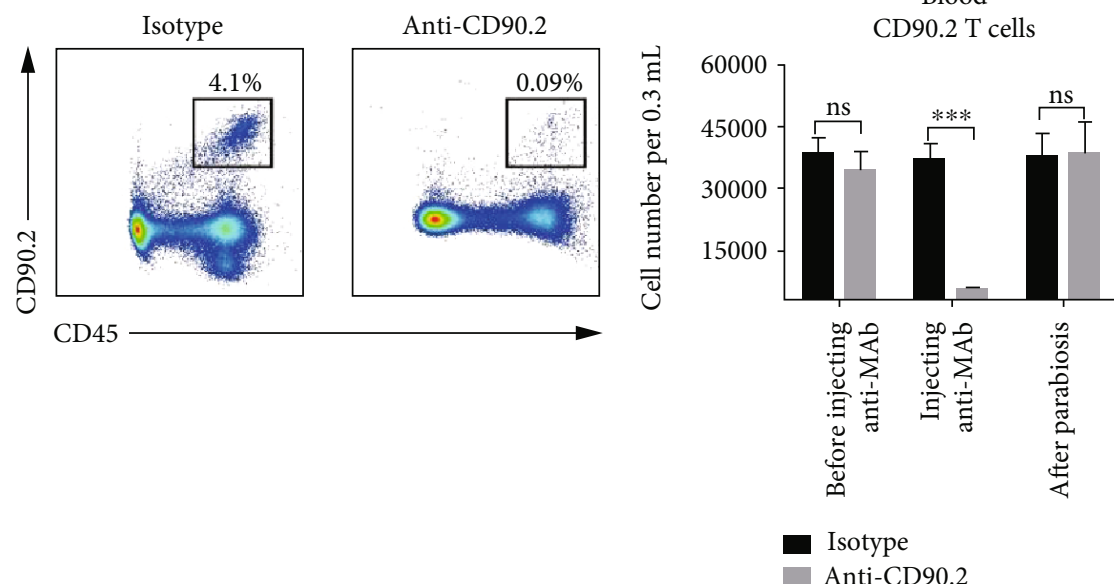

(b)

(c)

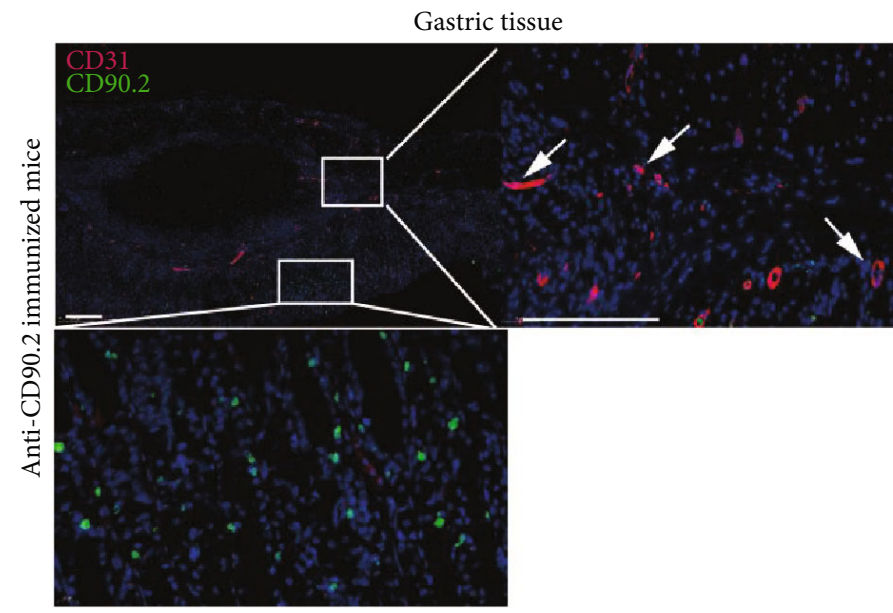

\begin{tabular}{lccc}
\hline Group & Left partner & Right partner & H. felis challenge \\
\hline 1 & Immunized WT & Isotype immunized WT & Right \\
2 & Immunized WT & Anti-CD90.2 immunized WT & Right \\
3 & Naive WT & Naive WT & Right
\end{tabular}

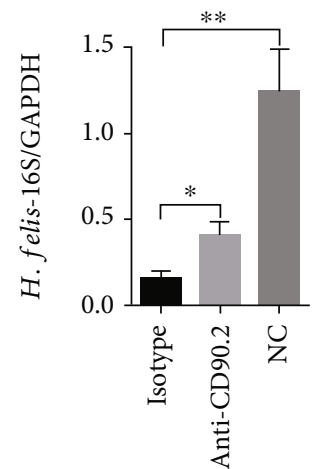

(d)

(e)

Figure 2: Perivascular lymphocyte clusters contributed to reduction of colonization of $H$. felis in gastric mucosa of mice. The depletion of perivascular lymphocyte clusters and a parabiosis mice model was performed as follows: (a) all mice were performed with laparotomy to access the stomach and injected with alum-based vaccine into GSL. At day 28, these immunized mice were divided into two groups. For one group, half of the mice were administered with anti-CD90.2 antibody and cohoused with the other half. At day 42, the anti-CD90.2 antibody-treated mice and the other mice not injected with an antibody were surgically jointed. At day 56, the indicated partner was infected with $\mathrm{H}$. felis. Another group was treated the same way but used IgG2b isotype. After the later challenge on day 3 , those mice were harvested and analyzed. (b, c) CD90.2 cells of the blood from indicated partner mice were analyzed by flow cytometry. Representative FACS plots were shown. (d) Frozen sections of gastric tissue from IgG2b isotype and CD90.2 antibody-treated mice were stained with antibodies against CD31 (red) and CD90.2 (green) and DAPI (blue). Scale bars: $200 \mu \mathrm{m}$. (e) Mice were performed as indicated in the table; 3 days later, the mRNA level of $H$. felis-16S was detected by QRT-PCR. Values are the mean \pm SD $(n=3)$. All indicated $P$ values were tested using the ANOVA analyses or t tests. ${ }^{* *} P<0.01 ;{ }^{* *} P<0.001$; n.s.: not significant. 


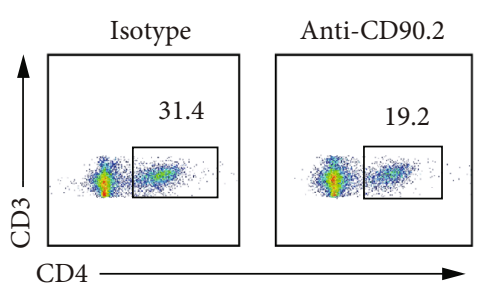

(a)

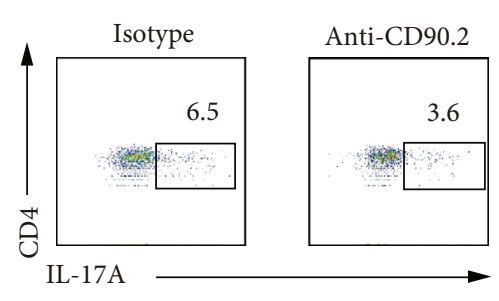

(d)

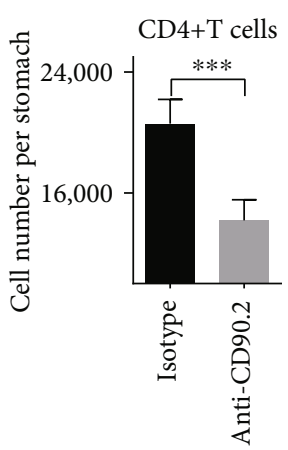

(b)

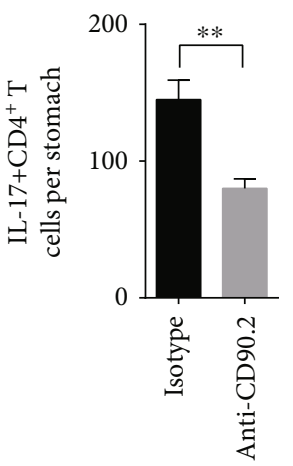

(e)

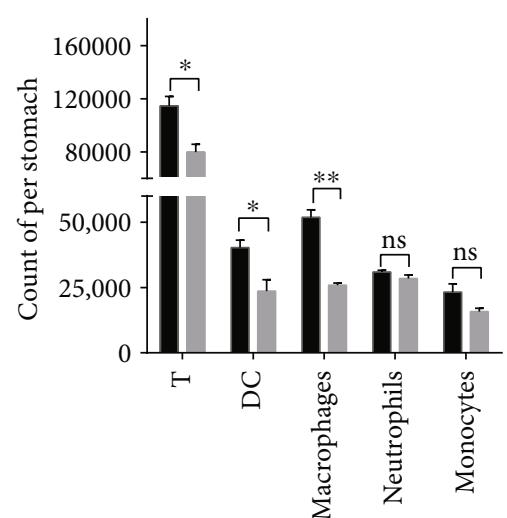

Isotype

Anti-CD90.2

(c)

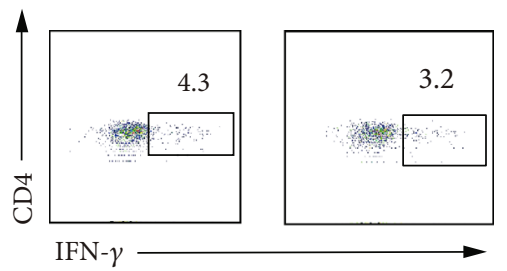

(f)

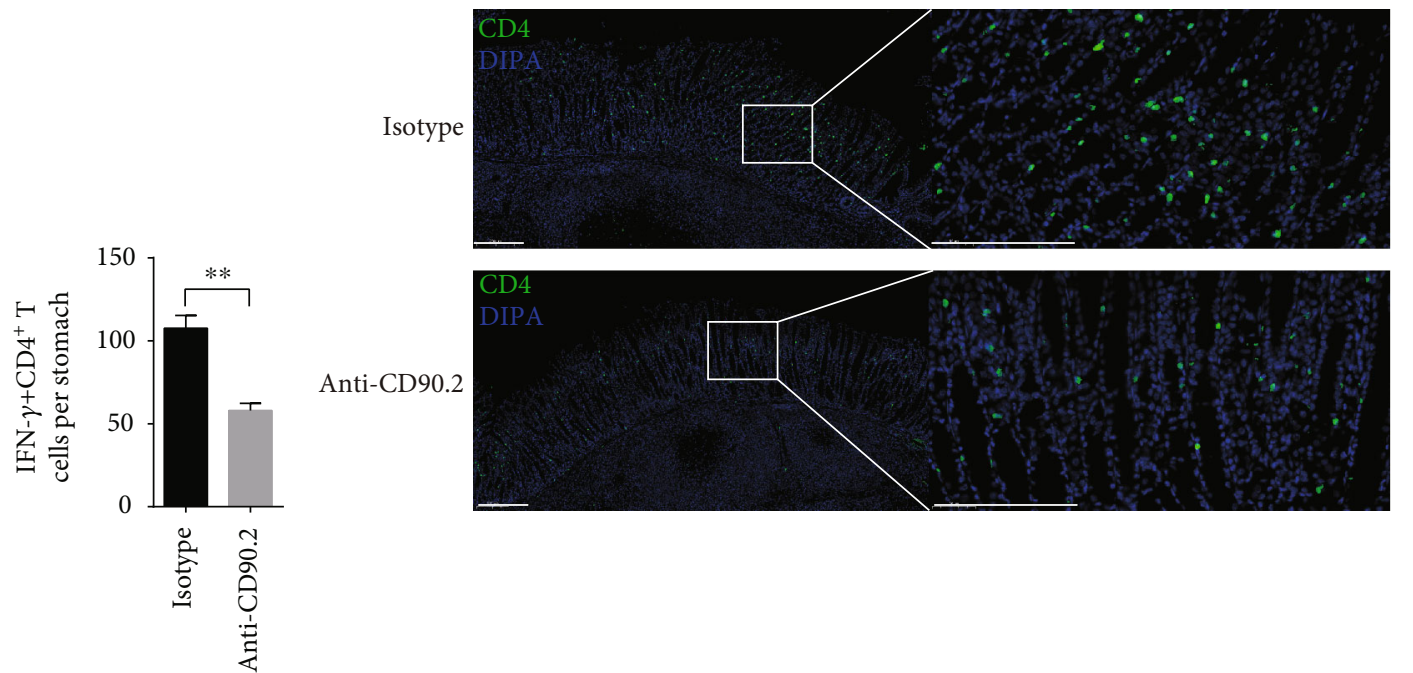

(g) (h)

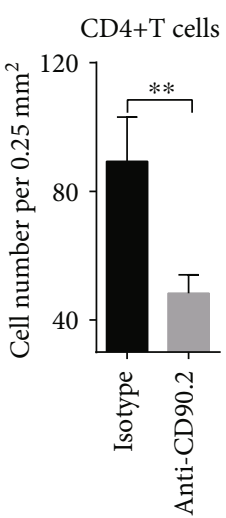

(i)

Figure 3: Perivascular lymphocyte clusters contributed to increased infiltrates of immune cells in gastric mucosa. Mice were administered as described in Figure 2(a) and the table of Figure 2(b). The single cells from gastric mucosa were analyzed by flow cytometry. The number of leukocytes (c), the percentage of total $\mathrm{CD} 4^{+} \mathrm{T}$ among $\mathrm{CD} 3^{+} \mathrm{T}$ cells, (a) and the number of total $\mathrm{CD} 4^{+} \mathrm{T}$ cells (b) were shown. (d-g) Single cells from gastric mucosa were stimulated with $1 * 10^{6}$ naïve CFSE-labeled splenocytes that were preloaded with CCF, and then the percentage and number of IFN- $\gamma / \mathrm{IL}-17$-producing $\mathrm{CD}^{+} \mathrm{T}$ cells were detected. (h) Frozen sections of gastric tissue from the indicated partner challenged with $H$. felis were stained with antibodies against CD4 (green), and nuclei were treated with DAPI (Blue). Scale bars indicated $200 \mu \mathrm{m}$. (i) Counts of CD4 cells per $0.25 \mathrm{~mm}^{2}$ were randomly chosen and calculated. Values are the mean \pm SD $(n=3)$. All indicated $P$ values were tested using the ANOVA analyses or t tests. ${ }^{*} P<0.05$; ${ }^{* *} P<0.01$; n.s.: not significant. 

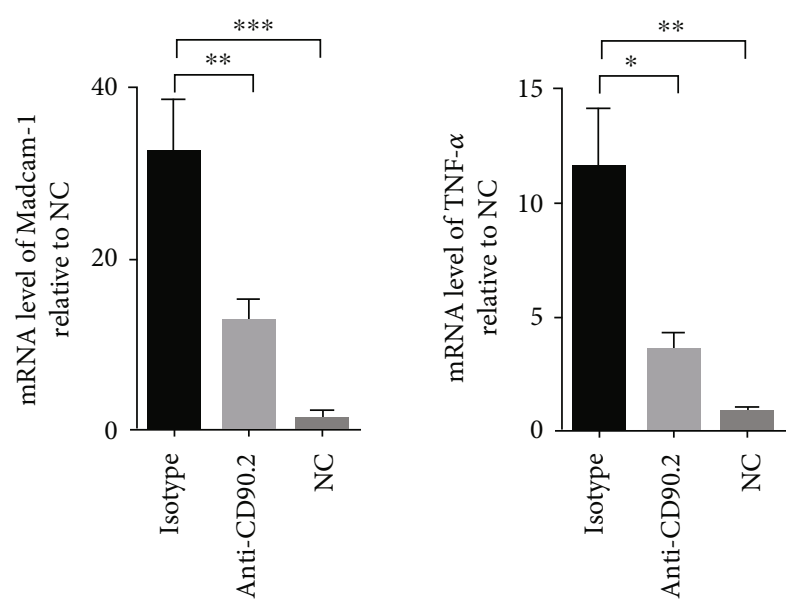

(a)

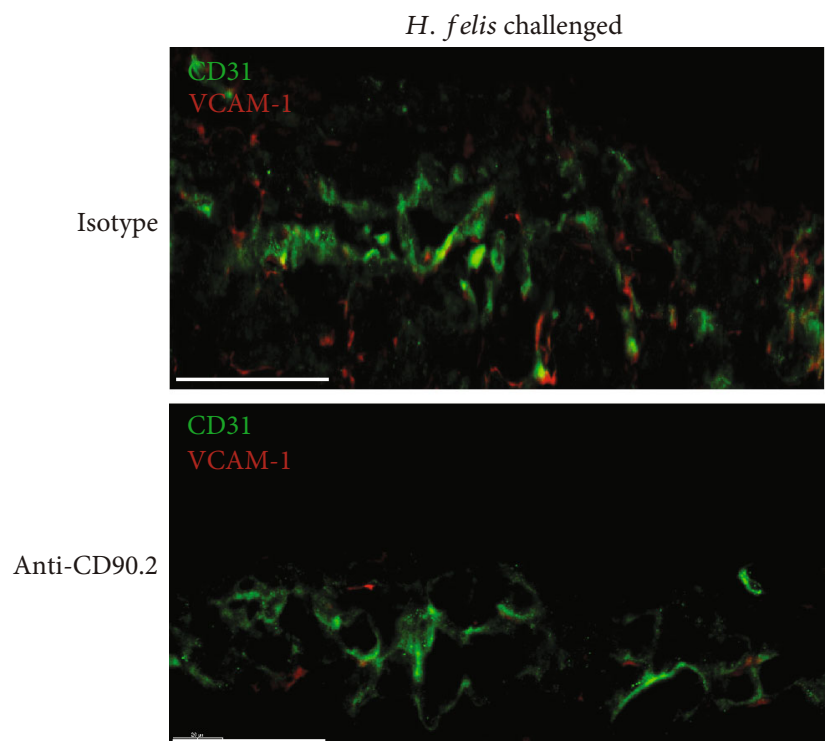

(b)

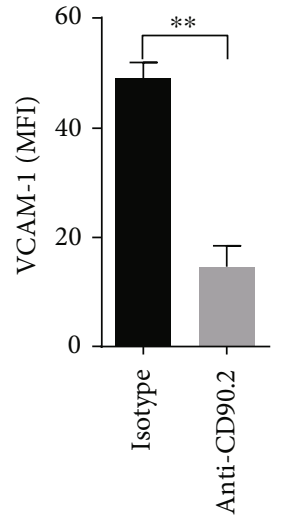

(c)

Figure 4: Perivascular lymphocyte clusters might increase the expression of VCAM-1, Madcam-1, and TNF- $\alpha$. Mice were treated as indicated in Figure 2(a). (a) The expression level of Madcam-1 and TNF- $\alpha$ in the gastric tissue of the indicated partner mice challenged with $H$. felis was analyzed by qRT-PCR. (b) Frozen sections of gastric tissue from the indicated partner challenged with $H$. felis were stained with antibodies against CD31 (green) and VCAM-1 (red), and nuclei were treated with DAPI (blue). Scale bars indicated $50 \mu \mathrm{m}$. (c) Mean fluorescence intensity (MFI) of VCAM-1 expression on $\mathrm{CD} 31^{+}$vessels was determined. Values are the mean $\pm \mathrm{SD}(n=3)$. All indicated $P$ values were tested using the ANOVA analyses or $\mathrm{t}$ tests. ${ }^{* *} P<0.01,{ }^{* * *} P<0.001$, and ${ }^{* * * *} P<0.0001$; n.s.: not significant.

clusters in recall responses. Subsequently, we focused on the correlation between colonization of $H$. felis and perivascular lymphocyte clusters and demonstrated that perivascular lymphocyte clusters facilitated gastric mucosal infiltration of proinflammatory immune cells and sIgA antibody upon $H$. felis infection.

In our previous study, we have established an in situ vaccination mouse model that harbored a $\mathrm{CD} 4^{+} \mathrm{T}_{\mathrm{RM}}$ pool in the stomach [6]. In the post vaccination acute phase, various immune cells were expanded and infiltrated into the vaccination region along with elevated levels of CXCL10 and CCL5
[6]. After resolution of inflammation, our data showed that abundant new vessels had emerged in the vaccinated region. Furthermore, we found that distinguished from native vessels, the new vessels were accompanied by a perivascular lymphocyte cluster. A recent study in the context of dermal perivascular leukocyte clusters suggests that a structure of perivascular DC-T cells clusters forms at the antigenchallenged site [9]. The DC clusters are followed by T cell accumulation around postcapillary venules and serve as the antigen-presentation sites for reactivation of effector $\mathrm{T}$ cells in the skin [9]. In comparison, we found in our models that 


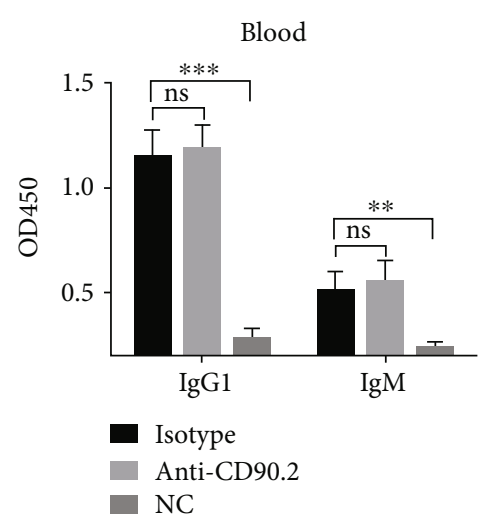

(a)

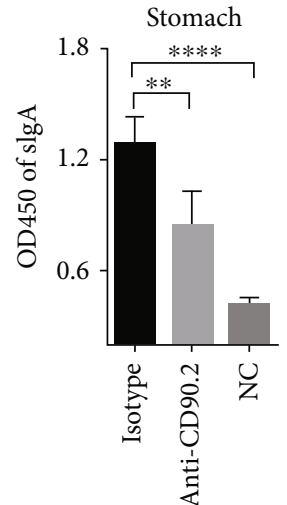

(b)

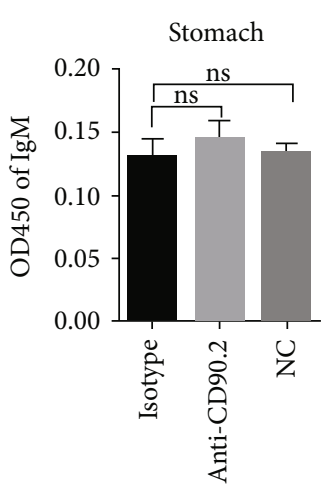

(c)

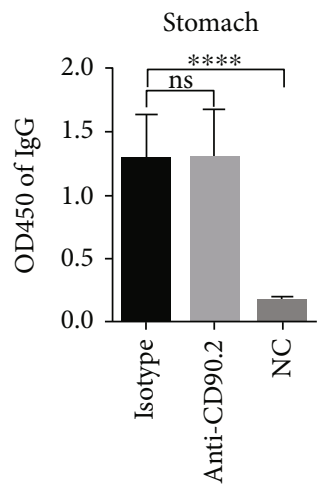

(d)

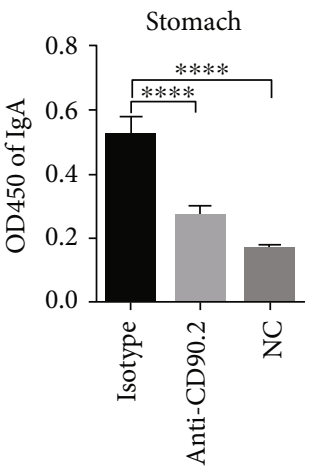

(e)

FIGURE 5: Perivascular lymphocyte clusters enhanced antigen-specific sIgA antibody response. All mice were performed with laparotomy to access the stomach and injected with alum-based vaccine into GSL. At day 28, these immunized mice were divided into two groups. For one group, half of the mice were administered with anti-CD90.2 antibody and cohoused with the other half. At day 42, the anti-CD90.2 antibodytreated mice and the other mice not injected with an antibody were surgically jointed. At day 56, the indicated partner was infected with $H$. felis. Another group was treated the same way but used IgG2b isotype. (a,b) After the later challenge on day 3, those mice were harvested and analyzed. The antigen-specific antibodies from the blood or gastric tissue were measured using ELISA. (c-e) After the later challenge at day 7 , those mice were harvested and analyzed. The antigen-specific IgM, IgG, and IgA from gastric tissue were measured using ELISA. The absorbance value was detected at $450 \mathrm{~nm}$ using a plate reader. Values were the mean $\pm \mathrm{SD}(n=3)$. All indicated $P$ values were tested using the ANOVA analyses or t tests. ${ }^{*} P<0.05,{ }^{* *} P<0.01$, and ${ }^{* * *} P<0.001$.

$\mathrm{CD}^{+}$and $\mathrm{CD}^{+} \mathrm{T}$ cells rather than $\mathrm{DCs}$ were the main subsets present within the perivascular lymphocytes.

In the study, the parabiosis mouse model and antiCD90.2 antibody depletion experiment demonstrated that depletion of perivascular lymphocyte clusters reduced the level of antigen-specific sIgA in the stomach, while no difference was seen in the level of IgG1 and IgM in the blood. The result suggested that perivascular lymphocyte clusters are related to mucosal sIgA response but not systemic antibody response. A recent research showed that $\mathrm{CD} 4^{+} \mathrm{T}$ cells help promote the access of antibody to neuronal tissue by triggering vascular permeability [23]. Proinflammatory cytokines act on endothelial cells to induce tight junction remodeling and increase permeability [24]. A similar result was observed, where the expression level of TNF- $\alpha$ correlated with perivascular lymphocyte clusters, which may facilitate mucosal infiltration of sIgA.

The stomach is a distinct organ that is inhabited by a very few native lymphocytes. Recruitment of circulating immune cells, such as Th1/Th17 cells and myeloid phagocytes, immediately is of utmost importance for inducing optimal anti- $H$. pylori response $[22,25,26]$. We found here that the perivascular lymphocyte clusters were "expressways" that allowed immune cell emigration into infected gastric mucosa. Depletion of perivascular lymphocyte clusters significantly reduced mucosal infiltration of DCs, macrophages, total T cells, and antigen-specific Th1/Th17 cells. Next, we investigated the correlation between migration of immune cells and perivascular lymphocyte clusters. Our data showed that mucosal infiltration of lymphocytes including total $\mathrm{T}$ cells and antigen-specific Th1/Th17 cells was expanded. It is noteworthy that lymphocytes generally permeate across the vessels by expressing VLA-4, which binds to endothelial VCAM-1 and alternatively spliced fibronectin (4). In a psoriatic skin, migration of lymphocytes into a psoriatic dermal skin is mediated by specific lymphocyte-dermal endothelial interactions [27]. This indicates that adhesion molecule or lymphocyte-endothelial interaction contributes to migration of lymphocytes. Consistent with the view that perivascular accumulation of lymphocytes is related to VCAM-1 expression in a perivascular adventitial fibroblast model [28], our data also demonstrated that the levels of expression of 
VCAM-1, Madcam-1, and TNF- $\alpha$ were attenuated in the absence of perivascular lymphocyte clusters. It is plausible that immune cells migrated through the endothelium by interaction between integrin ligands and adhesion molecules such as VCAM-1- $\alpha 4 \beta 1$ and Madcam-1- $\alpha 4 \beta 7$ [29, 30]. Meanwhile, recent studies found that local $\mathrm{CD}^{+} \mathrm{T}_{\mathrm{RM}}$ do not only facilitate the access of circulating memory $\mathrm{T}$ cells to the site of virus reinfection in the genital tract but also secretes cytokines to trigger innate immunity in an IFN- $\gamma$ VCAM-1 axis [31, 32]. As described above, we proposed that these increased adhesion molecules and cytokines contributed to the emigration of immune cells. Furthermore, $\mathrm{CD} 4^{+}$ $\mathrm{T}_{\mathrm{RM}}$ might secrete cytokines to trigger the accumulation of perivascular lymphocyte clusters in the context of our mouse model [6]. Our future study would explore the correlation between $\mathrm{CD}^{+} \mathrm{T}_{\mathrm{RM}}$ and perivascular lymphocyte clusters in the GSL-vaccination mouse model.

In some researches, an in situ immunization or infection mouse model was employed to explore the formation of $\mathrm{T}_{\mathrm{RM}}$ after the resolution of immunization or infection-induced inflammatory responses [1, 33]. However, new vessels emerged after the resolution of local inflammation, but their role on immune responses often has not been given attention. Interestingly, our study indicated that in situ gastric vaccination-induced local inflammation induced not only the establishment of a CD4 ${ }^{+} \mathrm{T}_{\mathrm{RM}}$ pool [6] but also the formation of new vessels with perivascular lymphocyte clusters, which contributed to mediating optimal immunity in recall response. These observations provide a new insight into the understanding of in situ vaccination-induced inflammatory response for the development of $H$. pylori vaccine. A concern is that GSL vaccination used in mice is not suitable for human, so our future study will look for a new approach to displace GSL vaccination. For example, a recent study shows that oral delivery of insulin targets the gastric mucosa by a self-orienting system [34]. This delivery system may be applicable for gastric mucosa-targeted vaccination.

\section{Conclusions}

The present study emphasized that in situ vaccinationinduced proinflammatory response induced not only the establishment of a $\mathrm{CD} 4^{+} \mathrm{T}_{\mathrm{RM}}$ pool but also the formation of perivascular lymphocyte cluster. Although the former provided prolonged protection, the latter also contributed to mediating optimal immunity by accelerating mucosal infiltration of immune cells and antibody response.

\section{Data Availability}

The data used to support the findings of this study are available from the corresponding author upon request.

\section{Conflicts of Interest}

The authors declare that they have no conflict of interest in this paper.

\section{Authors' Contributions}

YX, TX, and WL designed and conducted the project. WL and $\mathrm{CH}$ performed most statistical analysis and experiments. NX, AH, ZZ, SL, ZXZ, FY, and MF performed some experiments. WL and $\mathrm{CH}$ wrote the paper. All authors read and approved the final manuscript.

\section{Acknowledgments}

We would like to thank Editage (http://www.editage.com/) for English language editing. This work was supported by the National Natural Science Foundation of China (Nos. 81971562 and 81502970), the National Key R\&D Program of China (No. 2017YFD0400303), the Postgraduate Research \& Practice Innovation Program of Jiangsu Province, the Priority Academic Program Development (PAPD) of Jiangsu Higher Education Institutions, the Six Talent Peaks Project in Jiangsu Province (No. 2018-WSW-003), and the Fundamental Research Funds for the Central Universities (No. 2632020TD025).

\section{Supplementary Materials}

Supplementary Table 1: primer sequences of genes for quantitative RT-PCR assay. Supplementary Figure 1: immune cells emigrated towards the infected epithelium of the stomach through vessels in recall response. (Supplementary Materials)

\section{References}

[1] S. N. Mueller and L. K. Mackay, "Tissue-resident memory T cells: local specialists in immune defence," Nature Reviews Immunology, vol. 16, no. 2, pp. 79-89, 2016.

[2] H. Shin, "Formation and function of tissue-resident memory T cells during viral infection," Current Opinion in Virology, vol. 28, pp. 61-67, 2018.

[3] N. T. Bennett and G. S. Schultz, "Growth factors and wound healing: part II. Role in normal and chronic wound healing," American Journal of Surgery, vol. 166, no. 1, pp. 74-81, 1993.

[4] B. Norberg and L. Rydgren, "Lymphocyte migration through the walls of the post-capillary venules," Lymphology, vol. 11, no. 4, pp. 211-215, 1978.

[5] H. Song, X. Lv, J. Yang et al., "A novel chimeric flagellum fused with the multi-epitope vaccine CTB-UE prevents Helicobacter pylori-induced gastric cancer in a BALB/c mouse model," Applied Microbiology and Biotechnology, vol. 99, no. 22, pp. 9495-9502, 2015.

[6] W. Liu, Z. Zeng, S. Luo et al., "Gastric subserous vaccination with Helicobacter pylori vaccine: an attempt to establish tissue-resident CD4+ memory $\mathrm{T}$ cells and induce prolonged protection," Frontiers in Immunology, vol. 10, p. 1115, 2019.

[7] A. N. Makanya, R. Hlushchuk, and V. G. Djonov, "Intussusceptive angiogenesis and its role in vascular morphogenesis, patterning, and remodeling," Angiogenesis, vol. 12, no. 2, pp. 113-123, 2009.

[8] S. A. Eming, B. Brachvogel, T. Odorisio, and M. Koch, "Regulation of angiogenesis: wound healing as a model," Progress in Histochemistry and Cytochemistry, vol. 42, no. 3, pp. 115-170, 2007. 
[9] Y. Natsuaki, G. Egawa, S. Nakamizo et al., "Perivascular leukocyte clusters are essential for efficient activation of effector T cells in the skin," Nature Immunology, vol. 15, no. 11, pp. 1064-1069, 2014.

[10] M. P. Bevilacqua, "Endothelial-leukocyte adhesion molecules," Annual Review of Immunology, vol. 11, pp. 767-804, 1993.

[11] B. J. Rabquer, Y. Hou, F. del Galdo et al., "The proadhesive phenotype of systemic sclerosis skin promotes myeloid cell adhesion via ICAM-1 and VCAM-1," Rheumatology, vol. 48, no. 7, pp. 734-740, 2009.

[12] Y. Taooka, J. Chen, T. Yednock, and D. Sheppard, "The integrin alpha9beta1 mediates adhesion to activated endothelial cells and transendothelial neutrophil migration through interaction with vascular cell adhesion molecule-1," The Journal of Cell Biology, vol. 145, no. 2, pp. 413-420, 1999.

[13] R. R. Lobb and M. E. Hemler, "The pathophysiologic role of alpha 4 integrins in vivo," The Journal of Clinical Investigation, vol. 94, no. 5, pp. 1722-1728, 1994.

[14] M. Schlesinger and G. Bendas, "Vascular cell adhesion molecule-1 (VCAM-1)-An increasing insight into its role in tumorigenicity and metastasis," International Journal of Cancer, vol. 136, no. 11, pp. 2504-2514, 2015.

[15] Z. Tan, W. Liu, H. Liu et al., "Oral Helicobacter pylori vaccineencapsulated acid-resistant HP55/PLGA nanoparticles promote immune protection," European Journal of Pharmaceutics and Biopharmaceutics: official journal of Arbeitsgemeinschaft fur Pharmazeutische Verfahrenstechnik eV, vol. 111, pp. 3343, 2017.

[16] L. Guo, K. Liu, G. Xu et al., "Prophylactic and therapeutic efficacy of the epitope vaccine CTB-UA against Helicobacter pylori infection in a BALB/c mice model," Applied Microbiology and Biotechnology, vol. 95, no. 6, pp. 14371444, 2012.

[17] J. Sung, C. P. Sodhi, L. Voltaggio et al., "The recruitment of extra-intestinal cells to the injured mucosa promotes healing in radiation enteritis and chemical colitis in a mouse parabiosis model," Mucosal Immunology, vol. 12, no. 2, pp. 503-517, 2019.

[18] S. Luo, W. Liu, Z. Zeng et al., "Toxic adjuvants alter the function and phenotype of dendritic cells to initiate adaptive immune responses induced by oral Helicobacter pylori vaccines," Helicobacter, vol. 23, no. 6, p. e12536, 2018.

[19] S. Engelmann, M. Togni, A. Thielitz et al., "T cell-independent modulation of experimental autoimmune encephalomyelitis in ADAP-deficient mice," Journal of Immunology, vol. 191, no. 10, pp. 4950-4959, 2013.

[20] Z. Zeng, W. Liu, S. Luo et al., "Shape of gastrointestinal immunity with non-genetically modified Lactococcus lactis particles requires commensal bacteria and myeloid cells-derived TGF$\beta 1$," Applied Microbiology and Biotechnology, vol. 103, no. 9, pp. 3847-3861, 2019.

[21] T. G. Blanchard, F. Yu, C. L. Hsieh, and R. W. Redline, "Severe inflammation and reduced bacteria load in murine helicobacter infection caused by lack of phagocyte oxidase activity," The Journal of Infectious Diseases, vol. 187, no. 10, pp. 16091615, 2003.

[22] H. Sun, H. Yuan, R. Tan et al., "Immunodominant antigens that induce Th1 and Th17 responses protect mice against Helicobacter pylori infection," Oncotarget, vol. 9, no. 15, pp. 12050-12063, 2018.
[23] N. Iijima and A. Iwasaki, "Access of protective antiviral antibody to neuronal tissues requires CD4 T-cell help," Nature, vol. 533, no. 7604, pp. 552-556, 2016.

[24] C. T. Capaldo, A. E. Farkas, R. S. Hilgarth et al., "Proinflammatory cytokine-induced tight junction remodeling through dynamic self-assembly of claudins," Molecular Biology of The Cell, vol. 25, no. 18, pp. 2710-2719, 2014.

[25] K. A. Eaton, M. Mefford, and T. Thevenot, "The role of T cell subsets and cytokines in the pathogenesis of Helicobacter pylori gastritis in mice," Journal of Immunology, vol. 166, no. 12, pp. 7456-7461, 2001.

[26] S. J. Czinn and T. Blanchard, "Vaccinating against Helicobacter pylori infection," Nature Reviews Gastroenterology \& Hepatology, vol. 8, no. 3, pp. 133-140, 2011.

[27] R. Sackstein, V. Falanga, J. W. Streilein, and Y. H. Chin, "Lymphocyte adhesion to psoriatic dermal endothelium is mediated by a tissue-specific receptor/ligand interaction," The Journal of Investigative Dermatology, vol. 91, no. 5, pp. 423-428, 1988.

[28] A. M. S. Barron, J. C. Mantero, J. D. Ho et al., "Perivascular adventitial fibroblast specialization accompanies $\mathrm{T}$ cell retention in the inflamed human dermis," Journal of Immunology, vol. 202, no. 1, pp. 56-68, 2019.

[29] R. Alon, P. D. Kassner, M. W. Carr, E. B. Finger, M. E. Hemler, and T. A. Springer, "The integrin VLA-4 supports tethering and rolling in flow on VCAM-1," The Journal of Cell Biology, vol. 128, no. 6, pp. 1243-1253, 1995.

[30] D. Vestweber, "Adhesion and signaling molecules controlling the transmigration of leukocytes through endothelium," Immunological Reviews, vol. 218, pp. 178-196, 2007.

[31] J. M. Schenkel, K. A. Fraser, V. Vezys, and D. Masopust, "Sensing and alarm function of resident memory CD8(+) T cells," Nature Immunology, vol. 14, no. 5, pp. 509-513, 2013.

[32] J. M. Schenkel, K. A. Fraser, L. K. Beura, K. E. Pauken, V. Vezys, and D. Masopust, “T cell memory. Resident memory CD8 T cells trigger protective innate and adaptive immune responses," Science, vol. 346, no. 6205, pp. 98-101, 2014.

[33] A. Zaid, L. K. Mackay, A. Rahimpour et al., "Persistence of skin-resident memory T cells within an epidermal niche," Proceedings of the National Academy of Sciences of the United States of America, vol. 111, no. 14, pp. 5307-5312, 2014.

[34] A. Abramson, E. Caffarel-Salvador, M. Khang et al., “An ingestible self-orienting system for oral delivery of macromolecules," Science, vol. 363, no. 6427, pp. 611-615, 2019. 


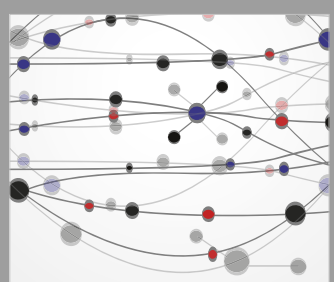

The Scientific World Journal
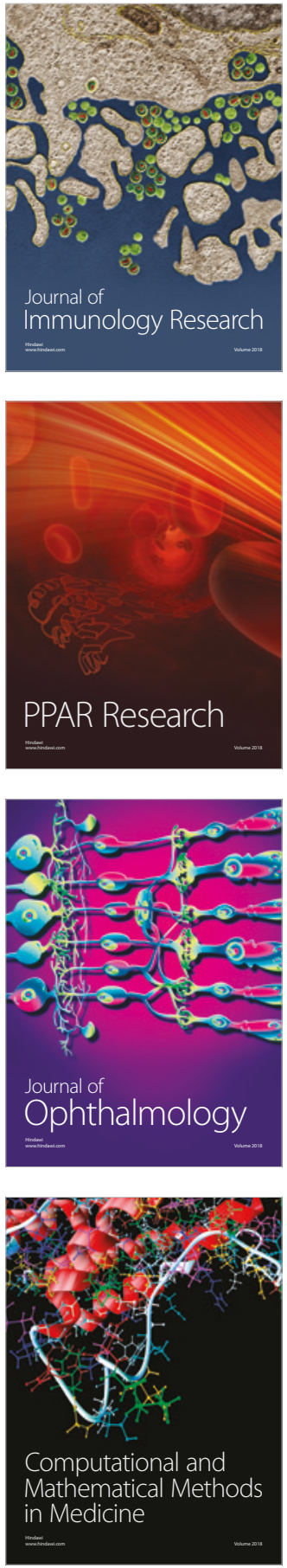

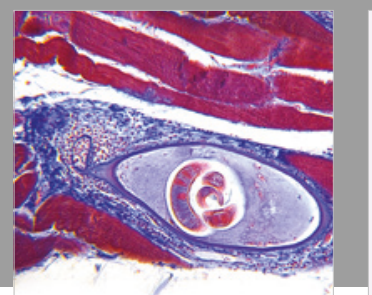

Gastroenterology Research and Practice

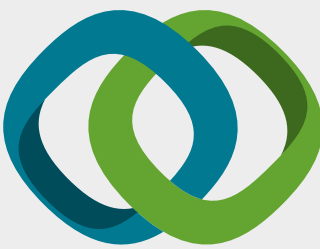

\section{Hindawi}

Submit your manuscripts at

www.hindawi.com
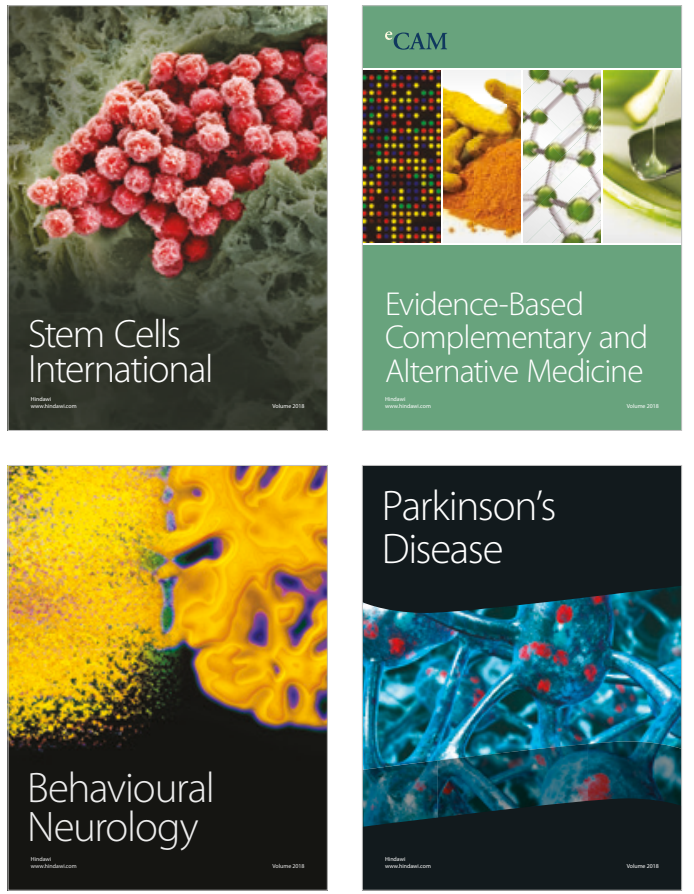

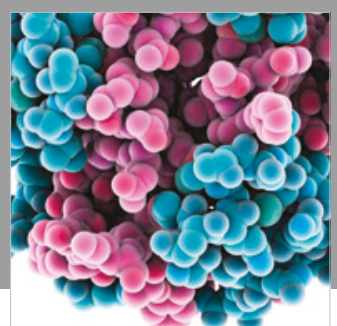

ournal of

Diabetes Research

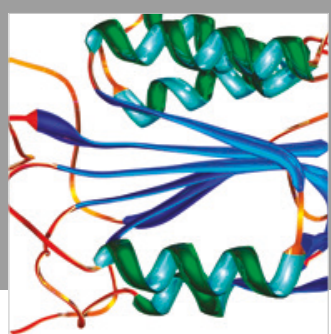

Disease Markers
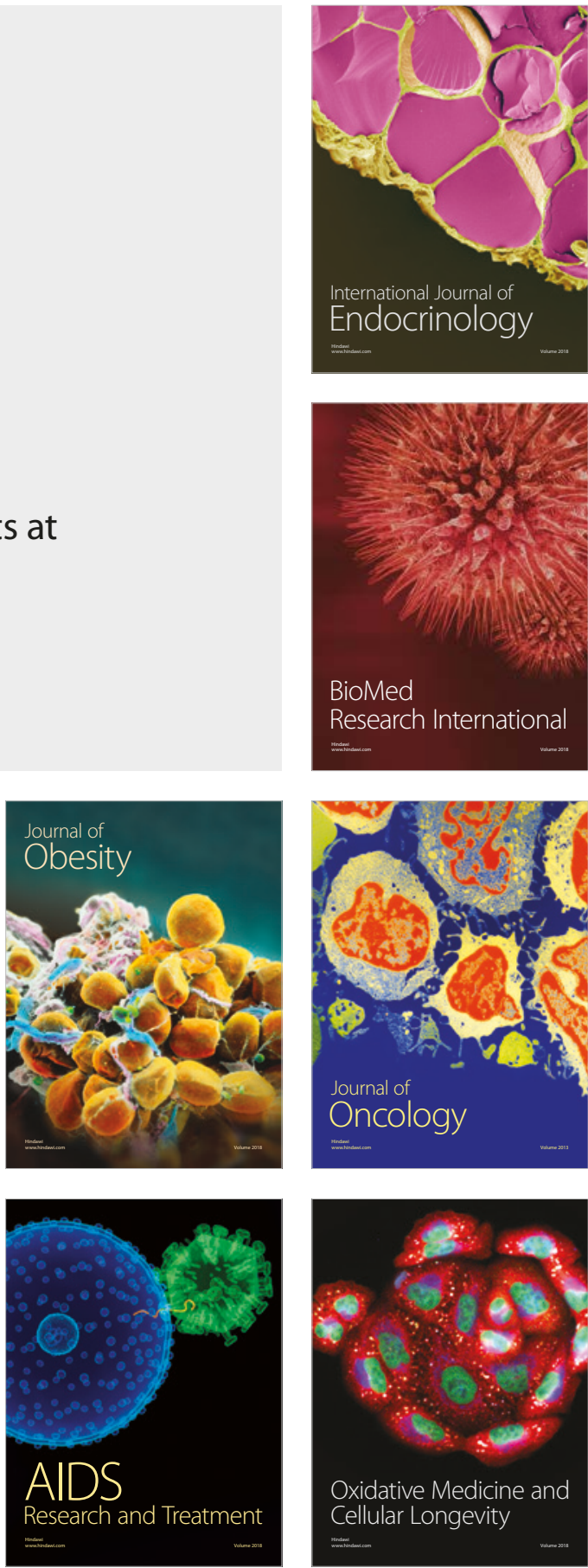